\title{
Enhanced Local Anesthetic Efficacy of Bioadhesive Ropivacaine Gels
}

\author{
Cheong-Weon Cho ${ }^{1}$, Jun-Shik $\mathrm{Choi}^{2}$ and Sang-Chul Shin ${ }^{3, *}$ \\ College of Pharmacy, ${ }^{1}$ Chungnam National University, Daejeon 305-764, ${ }^{2}$ Chosun University, Gwangju 501-759, \\ ${ }^{3}$ Chonnam National University, Gwangju 500-757, Republic of Korea
}

\begin{abstract}
In relieving local pains, ropivacaine has been widely used. In case of their application such as ointments and creams, it is difficult to expect their effects for a significant period of time, because they are easily removed by wetting, movement and contacting. Therefore, the new formulations that have suitable bioadhesion were needed to enhance local anesthetic effects. The effect of drug concentration and temperature on drug release was studied from the prepared $1.5 \%$ Carboxymethyl cellulose (CMC) (150 $\mathrm{MC}$ ) gels using synthetic cellulose membrane at $37 \pm 0.5^{\circ} \mathrm{C}$. As the drug concentration and temperature increased, the drug release increased. A linear relationship was observed between the logarithm of the permeability coefficient and the reciprocal temperature. The activation energy of drug permeation was $3.16 \mathrm{kcal} / \mathrm{mol}$ for a $1.5 \%$ loading dose. To increase the skin permeation of ropivacaine from $\mathrm{CMC}$ gel, enhancers such as saturated and unsaturated fatty acids, pyrrolidones, propylene glycol derivatives, glycerides, and non-ionic surfactants were incorporated into the ropivacaine-CMC gels. Among the enhancers used, polyoxyethylene 2-oleyl ether showed the highest enhancing effects. For the efficacy study, the anesthetic action of the formulated ropivacaine gel containing an enhancer and vasoconstrictor was evaluated with the tail-flick analgesimeter. According to the rat tail-flick test, $1.5 \%$ drug gels containing polyoxyethylene 2-oleyl ether and tetrahydrozoline showed the best prolonged local analgesic effects. In conclusion, the enhanced local anesthetic gels containing penetration enhancer and vasoconstrictor could be developed using the bioadhesive polymer.
\end{abstract}

Key Words: Local anesthetics, Ropivacaine, Bioahhesive, Gel, Penetration enhancer, Tail flick anesthetic test

\section{INTRODUCTION}

Local anesthetics are often used to alleviate pain after surgery, medical procedures and dental pains (Smith et al., 1999). They are also used in the control of postoperative pain and in the therapy of chronic pain (Dahm et al., 2000) and can be used for regional control of major pain. In relieving local pains, ropivacaine, one of local anesthetics belonging to the amide group, has been used. Of many drug delivery systems, percutaneous drug delivery has some advantages of providing the controlled delivery of the drug for an extended period of time.

One of these local anesthetic drugs is ropivacaine, which is characterized by its long action and high therapeutic power (Ritchie et al., 1989). There is a substantial population with intractable pain that is not responsive to opioids that require non-opioid agents, including local anesthetics (Dahm et al., 2000; Tsai et al., 2000). Ropivacaine plays a valuable role in the overall management of surgical and postoperative pain

www.biomolther.org

Open Access DOI: 10.4062/biomolther.2011.19.3.357

pISSN: 1976-9148 elSSN: 2005-4483

Copyright $\odot 2011$ The Korean Society of Applied Pharmacology associated with dental care (Moore, 2007). Of many drug delivery systems, percutaneous drug delivery can provide controlled delivery of drugs. However, in case of their application such as ointments and creams, it is difficult to expect their effects for a significant period of time, because they are easily removed by wetting, movement and contacting. Therefore, the new formulations that have suitable bioadhesion were needed to enhance local anesthetic effects.

Carboxymethyl cellulose (CMC) is used to control drug release from several pharmaceutical systems because of its non-toxic nature, easy compression, swelling properties, and accommodation of high levels of drug (Nairn, 2000). To formulate bioadhesive gels, we compared the viscosity and bioadhesive forces of $\mathrm{CMC}$, as well as drug release as a function of temperature and drug concentration. To increase the skin permeation of ropivacaine from the CMC gels, enhancers such as saturated and unsaturated fatty acids, pyrrolidones, propylene glycol derivatives, glycerides, and the non-ionic surfactants were incorporated in the ropivacaine-CMC gels. The local an-

Received Mar 26, 2011 Revised Jun 14, 2011 Accepted Jun 15, 2011

\section{* Corresponding Author}

E-mail: shinsc@chonnam.ac.kr

Tel: +82-62-530-2924, Fax: +82-62-530-2949 
esthetic effects of the formulated ropivacaine-CMC gels containing polyoxyethylene 2-oleyl ether was evaluated in the tail flick anesthetic test.

The objective of this study was to develop a bioadhesive gel formulation showing the enhanced local anesthetic efficacy.

\section{MATERIALS AND METHODS}

\section{Materials}

Ropivacaine hydrochloride was supplied from Dong-Woo Co. Ltd. (Korea). Carboxymethyl cellulose sodium was obtained from Bolak Co. Ltd. (Korea). Polyethylene glycol, lauric acid, oleic acid, caprylic acid, myristic acid, linoleic acid, stearic acid, palmitic acid, 2-pyrrolidone, 1-methyl-2-pyrrolidone, polyoxyethylene-2-stearyl ether (Brij 72), polyoxyethylene-23lauryl ether (Brij 35) and polyoxyethylene-2-oleyl ether (Brij 92) were purchased from Sigma-Aldrich Co. (USA). Oleyl macrogol-6 glycerides, caprylocaproyl mcarogol-8 glycerides, propylene glycol laurate, and propylene glycol monolaurate were gift from Gattefose (France). Methanol was HPLC grade from J.T.Baker Inc. (USA). All reagents of analytical grade were used without further purification.

\section{Measurement of viscosity and bioadhesive strength}

The viscosity and bioadhesive force of various types of CMC were measured at $1.5 \% \mathrm{CMC}$ concentration, respectively. The viscosity was measured with an MV II at a shear rate of 1.8 $1 /$ s using rotary viscometer (Haake Co, Germany) and sample equilibration took approximately $45 \mathrm{sec}$. The viscosity of sample was then determined by multiplying the observed reading. The bioadhesive capacity was determined by measuring the maximum detachment force using an Auto Peeling Tester (C.K. Trading, Korea). Cyanoacrylate adhesive was used to fix the intestinal mucosa to the upper and lower support. The CMC gel was placed on the both supports. Upon contact of the gel-intestine mucosa, a force was applied for five minutes. This procedure was performed at a speed of $150 \mathrm{~mm} / \mathrm{min}$ until the complete detachment of the components was achieved. The adhesion work was calculated as N (Newton force).

\section{Drug release from the $\mathrm{CMC}$ gels through cellulose membrane}

The in vitro release of ropivacaine from the CMC gels was examined by using the modified Franz diffusion cell. The diameter of the cell was $2 \mathrm{~cm}$, providing $3.14 \mathrm{~cm}^{2}$ effective constant area between the membrane and the bulk solution of 20 $\mathrm{ml}$. The flux of ropivacaine from the CMC gels was determined using the phosphate buffer solution $(\mathrm{pH} \mathrm{7.4)}$ as a receptor. The synthetic cellulose membrane was mounted on the receptor compartment of the diffusion cell.

Five grams of prepared CMC gels containing ropivacaine was placed in intimate contact with the cellulose membrane and the donor cap was covered with a parafilm and clamped. The sampling port was sealed with a parafilm to prevent the evaporation of the receptor medium. The receptor solution, phosphate buffer solution, was maintained at $37^{\circ} \mathrm{C}$ by a circulating water bath and stirred by a magnetic stirring bar. The donor compartment was maintained at ambient temperature. The effect of drug concentration on its release from the gels was studied according to drug concentration of $0.5,1,1.5,2$ and $2.5 \%(\mathrm{w} / \mathrm{w})$, and the effects of temperature on drug re- lease was performed at $27,32,37$ and $42^{\circ} \mathrm{C}$ by thermostated water bath. All samples $(20 \mathrm{ml})$ from the receptor compartment were withdrawn at predetermined intervals to maintain a sink condition, and immediately replaced by the same amount of fresh phosphate buffer solution.

\section{HPLC determination of ropivacaine}

Ropivacaine was assayed by HPLC method. The HPLC system was consisted with pump (Knauer, DE/K-120, USA), ultraviolet detector (Waters 484, USA), $\mathrm{C}_{18}$ column $(250 \times 4.6$ $\mathrm{mm}, 5 \mu \mathrm{m}$ ), degaser, and an integrator (D520A, Youngin scientific Co. Ltd. Korea). The mobile phase was composed with a mixture of methanol, water, phosphoric acid (30:70:0.1 $\mathrm{v} / \mathrm{v} / \mathrm{v})$. A flow rate of $1.0 \mathrm{ml} / \mathrm{min}$ yielded an operation pressure of $-1,000$ psi. The UV detector was operated at the wavelength of $220 \mathrm{~nm}$. Under these conditions, ropivacaine peak appeared at the retention time of $4.2 \mathrm{~min}$.

\section{Preparation of ropivacaine-CMC gels containing an enhancer}

1.5 grams of carboxymethyl cellulose sodium was dissolved in hot water to make $35 \mathrm{~g} .1 .5 \%$ ropivacaine and $5 \%$ enhancer were added with vigorous stirring to the above CMC solutions and water was added to make $100 \mathrm{~g}$.

\section{Skin preparation}

A male rat (Sprague Dawley rat strain) was sacrificed by snapping the spinal cord at the neck. The hair of abdominal area was carefully removed with an electric clipper. A square section of the abdominal skin was excised. After incision, the adhering fats and other visceral debris in the skin were carefully removed from the undersurface with tweezers. The excised skin was used immediately.

\section{Penetration of ropivacaine from the CMC gels through rat skins}

The drug permeation through rat skin from the $1.5 \%$ ropivacaine-CMC gel formulations was carried out at $37^{\circ} \mathrm{C}$ using phosphate buffer solution ( $\mathrm{pH} 7.4)$ as a receptor medium. The freshly excised full-thickness skin sample was mounted on the receptor site of the diffusion cell with the stratum corneum side facing upwards into the donor compartment and the dermal side facing downwards into the receptor compartment. Appropriate amount of gels was placed on the stratum corneum side and covered with round glass plate and clamped. Receptor medium, phosphate buffer solution $(\mathrm{pH} 7.4)$ was maintained $37^{\circ} \mathrm{C}$ by a circulating water bath. Total samples were withdrawn at predetermined time intervals and immediately replaced by an equal volume of fresh medium. Permeation quantities of ropivacaine were determined analyzed by HPLC at $220 \mathrm{~nm}$. Each data point represents the average of three determinations.

The cumulative amount of ropivacaine through the rat skin was plotted against time ( $\mathrm{min}$ ). A linear profile was observed for $2 \mathrm{~h}$ and the slope of the linear portion of the curve was determined by linear regression. The effectiveness of penetration enhancers was defined as the enhancement factor (EnF). EnF was calculated using the following equation: $\begin{aligned} \text { EnF }= & \text { (flux of ropivacaine-CMC gels containing enhancers }) \\ & /(\text { flux of the control })\end{aligned}$ 


\section{Tail flick anesthetic test}

Male Sprague-Dawley rats, 7-8 weeks old (270-300 g), were purchased from Daehan Laboratory Animal Research Co. (Choongbuk, Republic of Korea). The animals were housed (four or five per cage) in laminar flow cages maintained at $22 \pm 2^{\circ} \mathrm{C}$, a $50-60 \%$ relative humidity, and a $12: 12$ $\mathrm{h}$ light-dark cycle. The experiments were carried out in accordance with the "Guiding Principles in the Use of Animals in Toxicology" adopted by the Society of Toxicology (USA) in July 1989 and revised in March 1999. The Animal Care Committee of Chonnam National University (Gwangju, Republic of Korea) approved the study design and procedures.

The heat radiant tail flick assay, developed by D'Amour and Smith in the 1940s (D'Amour and Smith, 1941), is a commonly used experimental model for thermo-pain quantification. In this test, a rodent tail is exposed to a light source (radiant heat) and the latency of tail withdrawal from the heat source is recorded and analyzed. Depending on the experimental settings, the tail flick technique can be used to determine the basal nociception level, the analgesic effectiveness of pharmacological agents, and tolerance formation.

The tail flick assay remains a valuable method due to its simplicity, reproducibility, its relatively low variation, and the minimal requirement for apparatus. Moreover, a unique feature of tail flick versus other thermo-pain quantification methods, such as hot plate or Hargreaves, is that a spinally mediated simple reflex is primarily, if not exclusively, involved to produce the end-point detection of a flicking tail (Ossipov and Gebhart, 1996). Thus the absence of complex behavior (e.g., paw-licking in hot-plate test) renders the tail flick method advantageous because it avoids the confounding effects arising from scoring or interpreting more complicated behaviors. In some cases, the tail flick test can even be carried out in lightly anesthetized animals (Ness and Gebhart, 1986).

The rats were divided into four groups containing three rats each: control gel group, ropivacaine gel group, ropivacaine gel containing an enhancer group, ropivacaine gel containing enhancer and vasoconstrictor group.

The rat was fixed on a tail-flick-test apparatus (Tail flick anesthetic meter, Harvard Co. Ltd. USA) with the tail, $10 \mathrm{~cm}$ from its tip, exposed to heat from a projector lamp. A single control switch simultaneously activated the light and a timer and the timer stops automatically when tail flicks. The time interval between switching on the light to flick of the tail was recorded. A 50 sec cut-off time was used to avoid thermal injury. A dose of $50 \mathrm{mg}$ of drug gels was applied to the root of the tail on the midline. The tail flick anesthetic test was started after administration, and the test was done every 5 min until the duration time fell to control value.

The area under the effective curve $\left(\right.$ AUEC $\left._{0 \rightarrow 120 \mathrm{~min}}\right)$ from time zero to $120 \mathrm{~min}$ of the rat tail flick test curve was calculated using the linear trapezoidal rule. The efficacy factor in local anesthetic effects of ropivacaine after topical application of ropivacaine gel containing polyoxyethylene 2-oleyl ether was compared with that control gel not containing any additives. The efficacy factor (EfF) was calculated using the following equation:

\footnotetext{
$\mathrm{EfF}=($ AUEC of ropivacaine gels containing enhancer $)$ I (AUEC of the control gel)
}

\section{RESULTS AND DISCUSSION}

\section{Effects of CMC concentration on the bioadhesive force and viscosity}

The viscosity of various kinds of $100 \mathrm{MC}, 150 \mathrm{MC}$ and 300 MC CMC gels at $1.5 \%$ concentration was 485,916 and 1,790 cps, respectively. The bioadhesive force of $100 \mathrm{MC}, 150 \mathrm{MC}$ and $300 \mathrm{MC}$ CMC gels at $1.5 \%$ concentration was 0.024 , 0.046 and $0.083 \mathrm{~N}$, respectively. $150 \mathrm{MC}-\mathrm{CMC}$ gels showed the best bioadhesive forces. As the viscosity of the CMC gels increased, the bioadhesive forces increased (Fig. 1).

Among the various types of CMC, $100 \mathrm{MC}$ gel showed the highest viscosity and bioadhesive force. From these experiments, $100 \mathrm{MC}$ that showed the highest bioadhesive force was chosen as a bioadhesive gel formulation. To develop the new gel formulation that has suitable bioadhesion, the easy determination of viscosity could be used instead of bioadhesive force assessment.

\section{Effect of ropivacaine concentration on the drug release}

The effect of ropivacaine concentration on drug release across synthetic cellulose membrane was studied from the prepared CMC gels for 2 hour at $37 \pm 0.5^{\circ} \mathrm{C}$. The concentrations tested were $0.5,1,1.5,2$ and $2.5 \%$, respectively. The flux of ropivacaine from the gel formulations through synthetic cellulose membranes (Spectrapor ${ }^{\circledR}$ MW 12-14,000) for $2 \mathrm{hr}$ was shown in Fig. 2. As the concentration of ropivacaine from

\section{- Bioadhesive force $(\mathrm{N})$ ......... Viscosity (cps)}

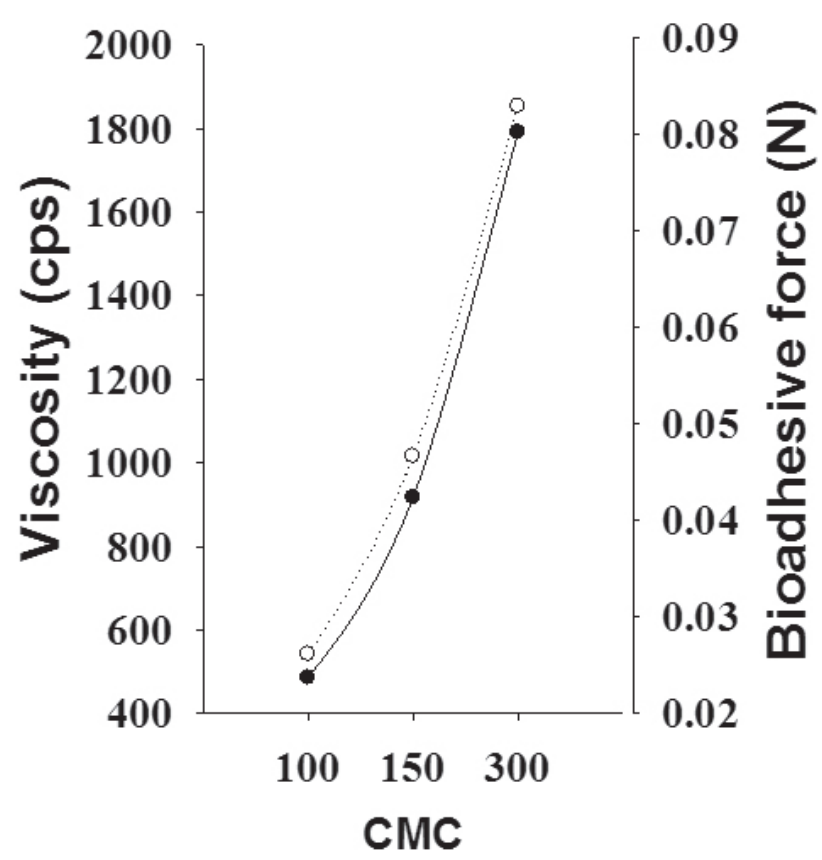

Fig. 1. Bioadhesive forces and viscosity of various kinds of $\mathrm{CMC}$ at $1.5 \%$ concentration. The viscosity was measured with an MV II at a shear rate of $1.81 / \mathrm{s}$ using rotary viscometer (Haake Co, Germany). The bioadhesive capacity was determined by measuring the maximum detachment force using an Auto Peeling Tester (C.K. Trading, Korea). 


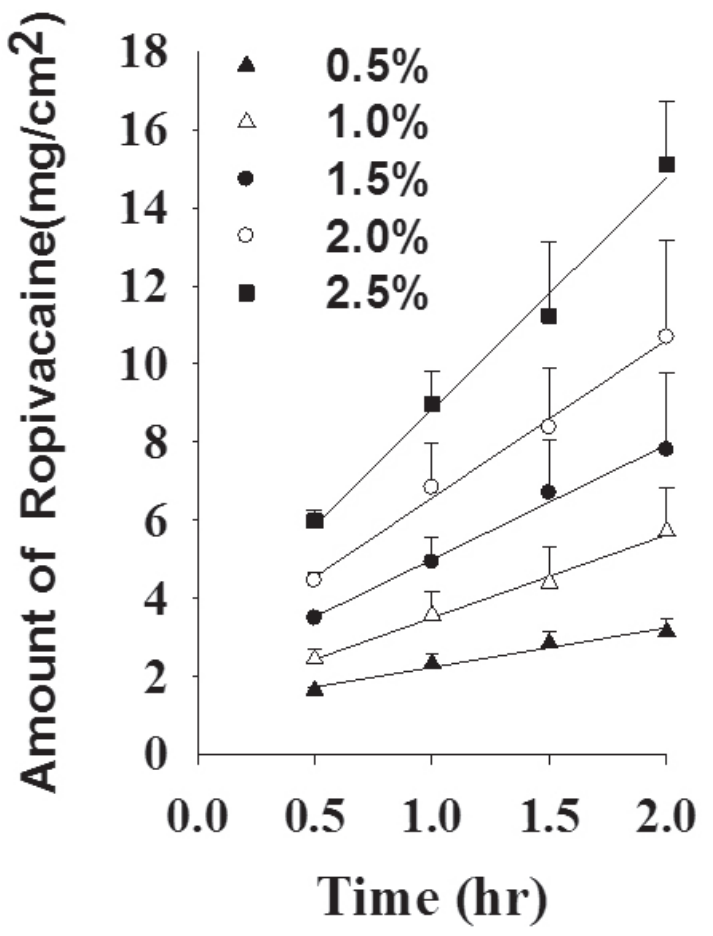

Fig. 2. Effect of ropivacaine concentration on drugs release through cellulose membrane from the CMC gels. The effect of ropivacaine concentration on drug release across synthetic cellulose membrane was studied from the prepared CMC gels for 2 hour at $37 \pm 0.5^{\circ} \mathrm{C}$. The concentrations tested were $0.5,1,1.5,2$ and $2.5 \%$, respectively.

the gels increased, drug release increased. As shown in Fig. 2, the permeation of drug followed Fick's law and exhibited concentration-dependent passive diffusion. Concerning the viscosity and appearance of the gel, $1.5 \%$ concentration of ropivacaine was chosen as an anesthetic gel formulation.

\section{Effect of temperature on drug release}

The effect of temperature on drug release from the 1.5\% ropivacaine gel formulations was evaluated at $27,32,37$ and $42^{\circ} \mathrm{C}$. All experiments were carried out at least in triplicate. The temperature dependency of drug release as a function of time was shown in Fig. 3.

A linear relationship was observed between the logarithm of the permeability coefficient $(P)$ and the reciprocal temperature (Fig. 3). The slope was used to calculate the activation energy $\left(E_{a}\right)$ for drug diffusion. The intercept was used to calculate the pre-exponential term.

The permeability coefficient is then defined by:

$$
\begin{aligned}
& P=\frac{\text { Flux }}{\text { Solubility }} \\
& P=P_{0} \cdot e^{-\frac{E_{a}}{R T}} \\
& \text { Log } P=\log P_{0}-\frac{E_{a}}{R \cdot 2.303 \cdot 1000} \cdot \frac{1000}{T} \\
& \text { Slope }=-\frac{E_{a}}{R \cdot 2.303} \cdot \frac{1}{1000}
\end{aligned}
$$

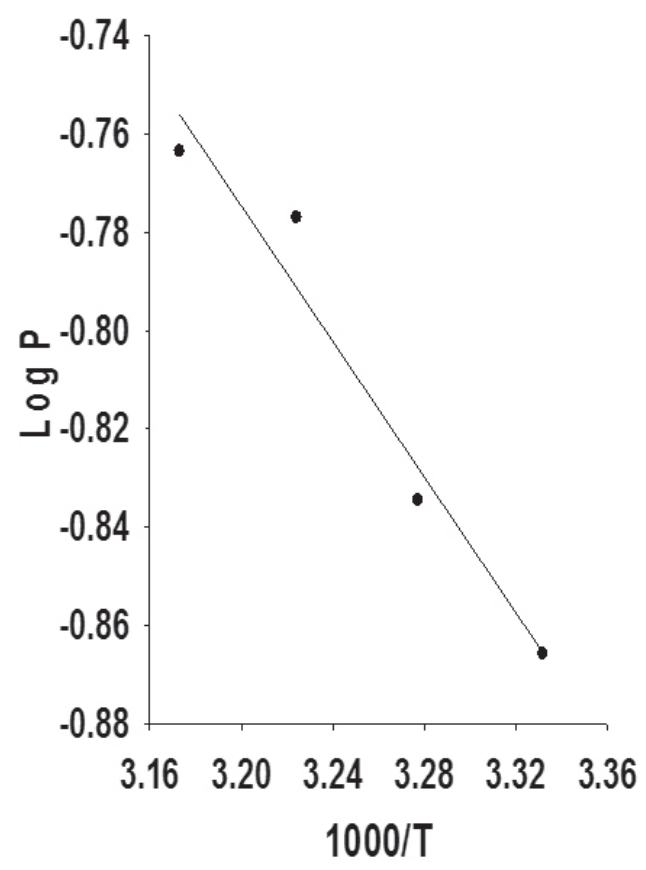

Fig. 3. Effect of temperature on drug release from the CMC gels containing $1.5 \%$ loading dose. The effect of temperature on drug release from the $1.5 \%$ ropivacaine-CMC gel formulations was evaluated at $27,32,37$ and $42^{\circ} \mathrm{C}$, respectively.

$$
\begin{aligned}
\text { Ea } & =- \text { Slope } \times R \times 2.303 \times 1000 \mathrm{cal} \\
& =- \text { Slope } \times 1.987 \times 2.303 \mathrm{kcal}
\end{aligned}
$$

As expected from Equation 5, the activation energy $\left(E_{a}\right)$ of drug permeation, which was calculated from the slope of log $P$ versus $1,000 / T$ plot, was $3.16 \mathrm{kcal} / \mathrm{mol}$ for a $1.5 \%$ loading dose. The observation indicates clearly that the release of drug from the gels is an energy-linked process (Miyazaki et al., 1984).

\section{Effect of enhancers on the permeation of ropivacaine across the rat skin}

To increase the skin permeation of ropivacaine from the CMC gel, various enhancers such as saturated and unsaturated fatty acids, pyrrolidones, propylene glycol derivatives, glycerides, and non-ionic surfactants, were incorporated in the ropivacaine-CMC gels. Skin is a complex, dynamic, layered organ that has many functions beyond its role as a barrier to the environment. The highly organized structure of the stratum corneum (SC) forms a barrier to substance penetration. To permeate the skin, the drug must diffuse through the hydrophilic and/or lipophilic environment of the SC to the deeper epidermal layers and to the dermis (Magnusson and Runn, 1999). However, the SC acts as a barrier and reduces skin permeability during transdermal drug delivery.

Penetration enhancers, accelerants, or promoters can interact with some components of skin to increase fluidity in the intercellular lipid lamellae, causing the SC to swell and/ or leach out structural components, thus increasing drug penetration through the barrier membrane (Magnusson and Runn, 1999). The penetration enhancers facilitate the absorption of penetrant through the skin by temporarily increasing the permeability of the skin. Some of the important penetration enhancers as classified by Sinha and Kaur (2000) are 
fatty acids, pyrrolidinones, alcohols, glycerides and polyoxyethylenes. These enhancers are divided into three groups as a manner of mechanism. One mechanism is increased skin/ vehicle partitioning of the drug. It was reported that the permeation enhancement of naphazoline by fatty acids could be caused by ion pair formation between drug and fatty acids, resulting in the increase of partitioning into the stratum corneum (Green and Hadgraft, 1988). A second likely mechanism of skin permeation enhancement is increased solvent transport into or across the skin. The results of increased solvent penetration may include increased drug solubility in the skin and increased skin penetration of the drug if the drug has a high affinity for the solvent (Yamada et al., 1987). The remaining proposed mechanism is increased drug solubility in the vehicle. Generally, acidic enhancers have been used to increase the solubility of basic drugs, and vice versa (Aungust and Rogers, 1980). Because of their widely different chemical structures, it is likely that the enhancers act by more than one mechanism and that their precise enhancer activity will depend on the physicochemical properties of the penetrant as well as the enhancer (Hadgraft and Walters, 1993). An ideal skin penetration enhancer is effective, nonirritating, and reversible (Barry and Bennett, 1987).

Fatty acids are currently receiving much attention as penetration enhancers (Tanojo et al., 1997; Oh et al., 1998). Fatty acids, by way of interactions with intercellular lipid domains, promote the skin permeation of drugs with a wide range of polarities. The efficacy of fatty acids is intrinsically linked to their structure, with differences evident between saturated and unsaturated forms and those of different hydrocarbon chain length (Kandimalla et al., 1999). Unsaturated fatty acids, particularly those of cis conformation and $\mathrm{C}_{18}$ chain lengths, are more effective enhancers than their saturated counterparts, promoting the permeation of penetrants such as naloxone (Aungust and Rogers, 1980) and flurbiprofen (Chi et al., 1995). When compared with a control, a saturated fatty acid group slightly increased the permeation rate. Among the saturated fatty acid group, myristic acid showed the highest permeation rate. Among the unsaturated fatty acids like oleic acid and linoleic acid, oleic acid showed significantly increased permeation of ropivacaine from $\mathrm{CMC}$ gel. In the case of fatty acids, a saturated fatty acid group increased the permeation rate more than an unsaturated fatty acid group (Choi and Shin, 2008).

Surfactants have been reported to enhance the permeability of drugs (Lopez et al., 2000; Shin et al., 2001; Shokri et al., 2001). They affect the permeability characteristics of several biological membranes, including skin (Florence et al., 1994; Lopez et al., 2000), and for this reason they can enhance the skin penetration of other compounds present in the formulation. In skin pre-treated with non-ionic surfactant, the SC was loosely layered and the intercellular spaces were wide (Shin et al., 2001; Choi and Shin, 2010). Therefore, in recent years, surfactants were used to enhance the permeation rates of several drugs. Among the non-ionic surfactants used, polyoxyethylene 2-oleyl ether (Brij 92) showed the greatest enhancing effects (Fig. 4).

Caprylocaproyl macrogol-glyceride (Labrasol) increases the passive transport of drug molecules. It exhibits high tolerance and low toxicity and included as a pharmaceutical excipient in European Pharmacopoeia (2008). Oleyl macrogol-6 glyceride (Labrafil) is a PEG derivative that is biocompatible and biodegradable (Gao et al., 1995) and used as a co-sur-

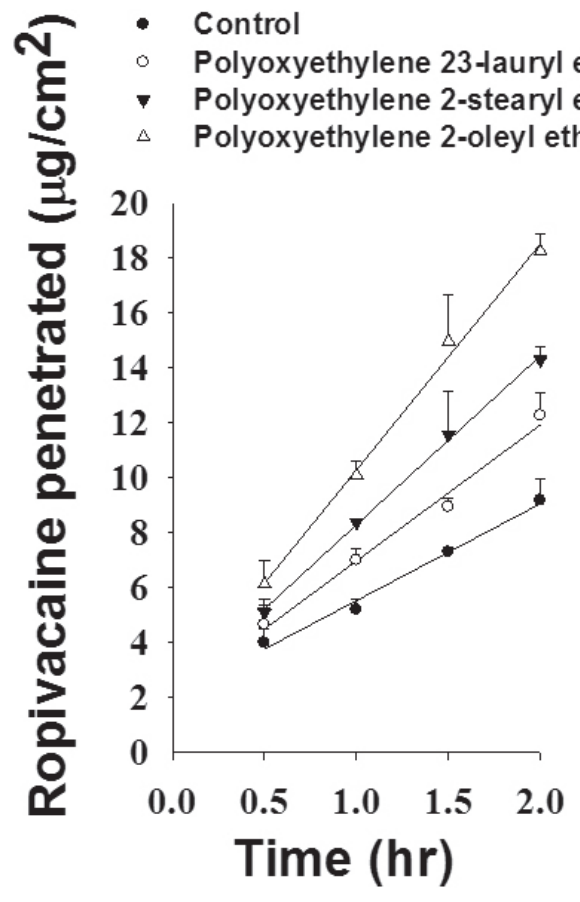

Fig. 4. Effect of the non-ionic surfactants on the penetration of ropivacaine from the $\mathrm{CMC}$ gels through the excised rat skin. The drug permeation through rat skin from the $1.5 \%$ ropivacaine-CMC gel formulations was carried out at $37^{\circ} \mathrm{C}$ using phosphate buffer solution $(\mathrm{pH} 7.4)$ as a receptor medium. Permeation quantities of ropivacaine were determined analyzed by HPLC at $220 \mathrm{~nm}$. Each data point represents the average of three determinations.

factant in pharmaceutical systems such as microemulsions. Among the glycerides, oleyl macrogol-6 glyceride shows a significant permeation rate for ropivacaine. Propylene glycol (PG) is widely used as a vehicle for penetration enhancers and permeates well through the human stratum corneum. PG readily permeates the skin and in so doing may carry the drug molecules across (Adrian and Barry, 2004).

Pyrrolidones have been used as penetration enhancers in human skin for hydrophilic and lipophilic permeants. In terms of mechanisms of action, the pyrrolidones partition well into the human corneum stratum. Within the tissue, they may act by altering the solvent nature of the membrane and pyrrolidones have been used to generate 'reservoirs' within skin membranes. Such a reservoir effect offers potential for sustained release of a permeant from the stratum corneum over an extended time period (Jungbauer et al., 2001).

The effects of penetration enhancers on the permeation rate of ropivacaine were determined by comparing the flux of drugs in the presence or the absence of enhancers.

$$
\begin{gathered}
\mathrm{EnF}=(\text { drug flux from the gel containing enhancer }) \\
\qquad / \text { (drug flux from the gel without enhancer })
\end{gathered}
$$

The enhancement factors $(E n F)$ of various enhancers are shown in Table 1. Among the enhancers tested such as the saturated, the unsaturated fatty acids, the pyrrolidones, the propylene glycol derivatives, the glycerides, and the non-ionic surfactants, polyoxyethylene 2-oleyl ether showed the highest enhancement. Enhancement factor of the ropivacaine gel 
Table 1. Enhancement factor of various enhancers.

\begin{tabular}{llll}
\hline \multicolumn{1}{c}{ Enhancer } & $\begin{array}{c}\text { Flux } \\
\left(\mu \mathrm{g} / \mathrm{cm}^{2} / \mathrm{hr}\right)\end{array}$ & EF \\
\hline Non ionic & Polyoxyethylene 23-lauryl ether & $4.960 \pm 0.06$ & 1.40 \\
surfactants & Polyoxyethylene 2-stearyl ether & $6.153 \pm 0.07$ & 1.74 \\
& Polyoxyethylene 2-oleyl ether & $8.245 \pm 0.10$ & 2.36 \\
Glycerides & Caprylocaproyl macrogol- & $7.247 \pm 0.10$ & 2.05 \\
& 8 glycerides & & \\
& Oleylmacrogol-6 glycerides & $6.308 \pm 0.09$ & 1.79 \\
Propylene & Propylene glycol mono & $5.779 \pm 0.06$ & 1.64 \\
glycols & caprylate & & \\
& Propylene glycol monolaurate & $5.811 \pm 0.06$ & 1.65 \\
& Propylene glycol laurate & $7.111 \pm 0.07$ & 2.01 \\
Pyrrolidones & 2-pyrrolidone & $4.054 \pm 0.03$ & 1.15 \\
& N-methyl-2-pyrrolidone & $6.327 \pm 0.07$ & 1.79 \\
& Polyvinyl-pyrrolidone & $5.053 \pm 0.05$ & 1.43 \\
Saturated & Stearic acid & $4.390 \pm 0.04$ & 1.24 \\
fatty acid & Caprylic acid & $6.100 \pm 0.06$ & 1.73 \\
& Lauric acid & $7.414 \pm 0.08$ & 2.10 \\
Unsaturated & Oleic acid & $4.773 \pm 0.05$ & 1.35 \\
\hline Each acid & Linoleic acid & $3.945 \pm 0.03$ & 1.12 \\
\hline
\end{tabular}

Each data point represents the average of three determinations.

Table 2. The comparison of $A \cup E C_{0 \rightarrow 120 ~ m i n}$ from the rat tail flick test for ropivacaine gels containing an enhancer or not

\begin{tabular}{lcc}
\hline & AUEC (sec $\cdot$ min) & $\begin{array}{c}\text { Efficacy } \\
\text { factor }\end{array}$ \\
\hline Control & $1,132.42 \pm 33.85^{*}$ & 1 \\
Ropivacaine gel & $2,066.78 \pm 43.73^{*}$ & 1.83 \\
$\begin{array}{l}\text { Ropivacaine gel containing } \\
\text { polyoxyethylene 2-oleyl ether }\end{array}$ & $2,654.84 \pm 71.49^{*}$ & 2.34 \\
$\begin{array}{l}\text { Ropivacaine gel containing } \\
\text { polyoxyethylene 2-oleyl ether }\end{array}$ & $2,960.34 \pm 155.3^{*}$ & 2.61 \\
and tetrahydrozoline & & \\
\hline
\end{tabular}

Each value represents the mean \pm S.D. $(n=3) .{ }^{*} p<0.05$.

containing polyoxyethylene 2-oleyl ether was 2.36 comparing the ropivacaine gel containing the no-enhancer.

\section{Tail-flick anesthetic test of ropivacaine gel containing an enhancer}

In percutaneous permeation studies, ropivacaine gel containing polyoxyethylene 2-oleyl ether showed the highest enhancing effects. Therefore, we tested the anesthetic effects of this preparation in a rat tail flick analgesic meter. Table 2 shows the $\mathrm{AUEC}_{0 \rightarrow 120 \mathrm{~min}}$ of the rat-tail flick test for anesthetic gel.

From the AUEC (Area Under the Efficacy Curve) of the rat tail flick test curve, the value of AUEC of ropivacaine gel containing polyoxyethylene 2-oleyl ether and tetrahydrozoline was $2,960.34 \mathrm{sec} \cdot \min$. The ropivacaine gel containing polyoxyethylene 2-oleyl ether was $2654.84 \mathrm{sec} \cdot \mathrm{min}$, while that not containing any additives was $2066.78 \mathrm{sec} \cdot \mathrm{min}$. The anes-

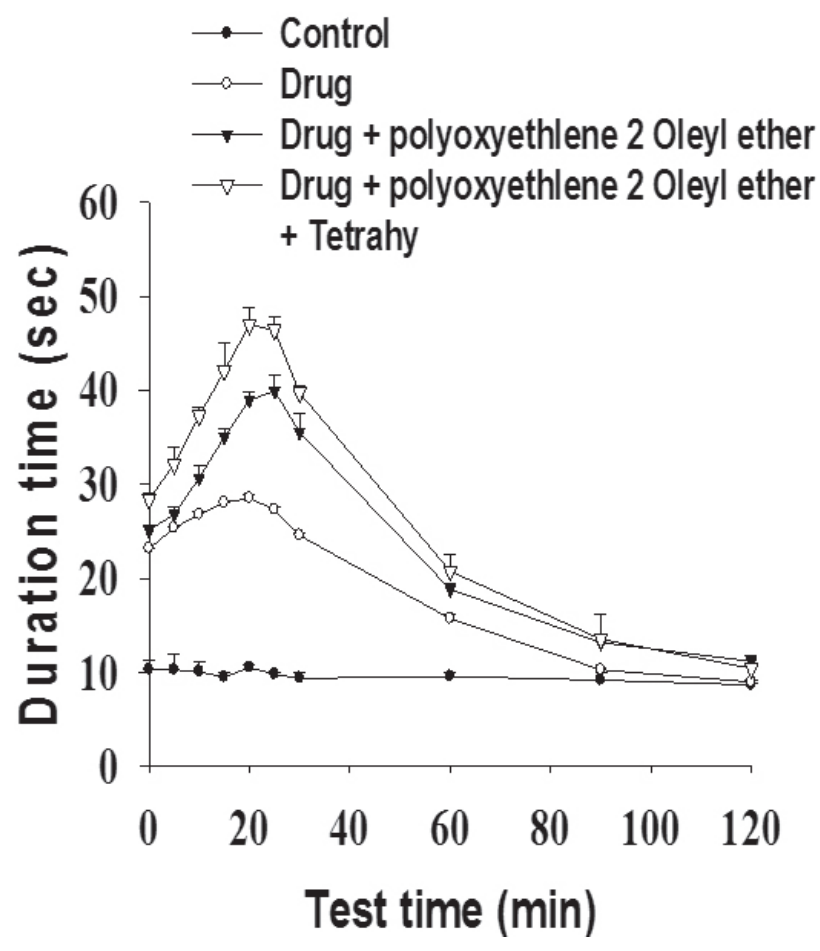

Fig. 5. Tail flick test of $1.5 \%$ ropivacaine gels. The rats were divided into four groups containing three rats each: control gel group, ropivacaine gel group, ropivacaine gel containing an enhancer group, ropivacaine gel containing enhancer and vasoconstrictor group. A dose of $50 \mathrm{mg}$ of drug gels was applied to the root of the tail on the midline. The tail flick anesthetic test using a tail-flick-test apparatus (Tail flick anesthetic meter, Harvard Co. Ltd. USA) was started after administration, and the test was done every 5 min until the duration time fell to control value. The area under the effective curve $\left(\right.$ AUEC $_{0 \rightarrow 120 \mathrm{~min}}$ ) from time zero to $120 \mathrm{~min}$ of the rat tail flick test curve was calculated using the linear trapezoidal rule.

thetic efficacy of ropivacaine gel containing polyoxyethylene 2-oleyl ether showed about 1.43-fold compared with that not containing any additives.

According to the rat tail flick test, $1.5 \%$ ropivacaine gel containing polyoxyethylene 2-oleyl ether showed the better prolonged anesthetic effects. The ropivacaine gel containing polyoxyethylene 2-oleyl ether showed prolonged, the highest anesthetic effects at $30 \mathrm{~min}$ and ropivacaine gel showed at $20 \mathrm{~min}$.

The combination with the vasoconstrictor, tetrahydrozoline, showed similar efficacy but an even longer anesthetic effect (Fig. 5). It is suggested that vasoconstrictor localized the ropivacaine concentration on applied area and showed longer anesthetic time.

The rate of drug release increased with increasing drug concentration and temperature. The anesthetic efficacy of ropivacaine gel containing polyoxyethylene 2-oleyl ether and tetrahydrozoline was 1.43 -fold higher than ropivacaine gel not containing additives. These results suggest that a topical gel formulation of ropivacaine containing polyoxyethylene 2-oleyl ether and tetrahydrozoline could be developed for enhanced local anesthetic effects. 


\section{REFERENCES}

Aungust, B. J. and Rogers, N. J. (1980) Comparison of the effects of various transmucosal absorption promoters on buccal insulin delivery. Int. J. Pharm. 53, 227-235.

Adrian, C. W. and Barry, B. W. (2004) Penetration enhancers. Adv. Drug. Deli. Rev. 56, 603-618.

Barry, B. W. and Bennett, S. L. (1987) Effect of penetration enhancers on the permeation of mannitol, hydrocortisone and progesterone through human-skin. J. Pharm. Pharmacol. 39, 535-546.

Choi, J. S. and Shin, S. C. (2008) Enhanced bioavailability by transdermal administration of pranoprofen gels containing an octanoic acid to rats. Biomol. Therap. 16, 210-214.

Choi, J. S. and Shin, S. C. (2010) Enhanced bioavailability of ambroxol by transdermal administration of the EVA matrix containing penetration enhancer in rats. Biomol. Therap. 18, 106-110.

Chi, S. C., Park, E. S. and Kim, H. (1995) Effect of penetration enhancers on flurbiprofen permeation through rat skin. Int. J. Pharm. 126, 267-274.

D'Amour, F. E. and Smith, D. L. (1941) A method for determining loss of pain sensation. J. Pharmacol. Exp. Ther. 72, 74-79.

Dahm, P., Lundborg, C., Janson, M., Olegard, C. and Nitescu, P. (2000) Comparison of $0.5 \%$ intrathecal bupivacaine with $0.5 \%$ intrathecal ropivacaine in the treatment of refractory cancer and noncancer pain conditions: results from a prospective, crossover, double-blind, randomized study. Reg. Anesth. Pain Med. 25, 480-487.

Florence, T., Tuker, I. G. and Walters, K. A. (1994) Interaction of nonionic alkyl and aryl ethers with membranes and other biological systems. In Structure Performance Relationships in Surfactants (M. J. Rosen, Ed.), pp. 189-207. ACS Symposium Series,

Gao, Z., Crowley, W. R., Shukla, A. J., Johnson, J. R. and Reger, J. F. (1995) Controlled release of contraceptive steroids from biodegradable and injectable gel formulations; in vivo evaluation. Pharm. Res. 12, 864-868.

Green, P. G. and Hadgraft, J. (1988) Facilitated transfer of cationic drugs across a lipoidal membrane by oleic acid and lauric acid. Int. J. Pharm. 37, 251-255.

Hadgraft, J. and Walters, K. A. (1993) Pharmaceutical Skin Penetration Enhancement. Marcel Dekker, New York. 3-4.

Jungbauer, F. H. W., Coenraads, P. J. and Kardaun, S. H. (2001) Toxic hygroscopic contact reaction to $\mathrm{N}$-methyl-2-pyrrolidone. Contact Dermatitis 45, 303-304.

Kandimalla, K., Kanikkannan, N., Andega, S. and Singh, M. (1999) Effect of fatty acids on the permeation of melatonin across rat and pig skin in vitro and on the transepidermal water loss in rats in vivo. J. Pharm. Pharmacol. 51, 783-790.

Lopez, A., Llinares, F., Cortell, C. and Herraez, M. (2000) Comparative enhancer effects of span 20 with Tween 20 and Azone on the in vitro percutaneous penetration of compounds with different lipophi- licities. Int. J. Pharm. 202, 133-140.

Magnusson, B. M. and Runn, P. (1999) Effect of penetration enhancers on the permeation of the thyrotropin releasing hormone analogue pGlu-3-methyl-His-Pro amide through human epidermis. Int. J. Pharm. 178, 149-159.

Miyazaki, S., Takeuchi, S., Yokouchi, T. and Takada, M. (1984) Pluronic F-127 gels as a vehicle for topical administration of anticancer agents. Chem. Pharm. Bull. 32, 4205-4208.

Moore, D. C. (2007) nLipid rescue from bupivacaine cardiac arrest: a result of failure to ventilate and maintain cardiac perfusion? Anesthesiology 106, 636-637.

Nairn, J. G. (2000) Solutions, emulsions, suspensions and extracts. In Remington: The Science and Practice of Pharmacy, (A. R. Gennaro, Ed.), 20th ed. pp. 745-747. Williams and Wilkins Inc., Lippincott, Philadelphia.

Ness, T. J. and Gebhart, G. F. (1986) Centrifugal modulation of the rat tail flick evoked by graded noxious heating of the tail. Brain Res. 386, 41-52.

Oh, S. Y., Jeong, S. Y., Park, T. G. and Lee, J. H. (1998) Enhanced transdermal delivery of AZT (Zidovudine) using iontophoresis and penetration enhancer. J. Cont. Rel. 51, 161-168.

Ossipov, M. H. and Gebhart, G. F. (1996) Adrenergenic influences on the modulation of nociception from the lateral nucleus of the rat. Brain Res. 384, 282-293.

Ritchie, J. M. and Greene, N. M. (1989) Aneste'sicos locales. In Las Bases Farmacolo'gicas de la Terape'utica (L. S. Goodman and A G. Gilman, Eds.), 7th ed. pp. 300-317. Editorial Me'dica Panamericana Madrid

Shin, S. C., Cho, C. W. and Oh, I. J. (2001) Effects of non-ionic surfactants as permeation enhancers towards piroxicam from the poloxamer gel through rat skins. Int. J. Pharm. 222, 199-203.

Shokri, J., Nokhodchi, A., Dashbolaghi, A., Hassan-Zadeh, D., Ghafourian, T. and Barzegar-Jalali, M. (2001) The effect of surfactants on the skin penetration of diazepam. Int. J. Pharm. 28, 99-107.

Sinha, V. R. and Kaur, M. P. (2000) Permeation enhancers for transdermal drug delivery. Drug Dev. Ind. Pharm. 26, 1131-1140.

Smith, D. W., Peterson, M. R. and DeBerard, S. C. (1999) Local anesthesia. Topical application, local infiltration, and field block. Postgraduate Medicine. 106, 57-60.

Tanojo, H., Bouwstra, J. A., Junginger, H. E. and Bodde, H. E. (1997) In vitro human skin barrier modulation by fatty acid: Skin permeation and thermal analysis studies. Pharm. Res. 14, 42-49.

Tsai, Y. C., Wang, L. K., Chen, B. S. and Chen, H. P. (2000) Homebased patient controlled epidermal analgesia with bupivacaine for patients with intractable herpetic neuralgia. J. Formos. Med. Assoc. 99, 659-662.

Yamada, M., Uda, Y. and Tanigawara, Y. (1987) Mechanism of enhancement of percutaneous absorption of molsidomine by oleic acid. Chem. Pharm. Bull. 35, 3399-3406. 